\title{
THE IMPACT OF PERSONAL FACTORS ON GIS ADOPTION IN CRISIS MANAGEMENT ORGANIZATIONS
}

\author{
Azita Asadi \\ Universiti Putra Malaysia \\ Serdang, Malaysia \\ E-mail: azita.asadi.UPM@gmail.com \\ Govindan Marthandan \\ School of Management \\ Multimedia University \\ Cyberjaya, Malaysia \\ E-mail:marthandan@mmu.edu.my \\ Majid FathiZahraei * \\ School of Management \\ Multimedia University \\ Cyberjaya, Malaysia \\ E-mail: majid.zahraei@gmail.com \\ Murali Raman \\ School of Management \\ Multimedia University \\ Cyberjaya, Malaysia \\ E-mail: murali.raman@mmu.edu.my
}

\begin{abstract}
The ever increasing crisis across the different regions of the world over the years led to increasing human vulnerability to various risks. For this reason, research has been geared towards identifying the impact of individual factors of managing risk at the various stages of crisis management; which are important benchmarks for assisting decision makers in putting in place the most feasible choices for minimizing human risks associated with crisis situations. In this paper, Analytic Network Process (ANP) is used to prioritize the impact of individual factors on Crisis Management. The study identified Attitude, Subjective norms, and Knowledge and experience as the three individual factors that influence Crisis Management. From the result of the study, it was shown that the prioritization of these factors varies between individuals and among stages.
\end{abstract}

Keywords: Disaster, Multiple Criteria Decision Making (MCDM), Attitude, Subjective norms, Geographic Information System (GIS).

\section{Introduction}

Increase in the destructive effects of crisis due to a surge in natural and man-made disasters has led to research interest in Crisis Management. According to EM-DAT (2013), the number of disasters rose to 19,063 from 1900 to 21 July 2012 and the 
consequence is, more than 38 million deaths and US\$2,4 thousand billion in cost of damages. As such, the number of people affected by the same types of hazards has increased significantly over the years . This issue relates to the growing world population. The Greenhouse effect on global warming and some technological and technical problems in industrial areas have caused a growing number of disasters, particularly from the 1960 s to the present .

Researchers and practitioners sometimes use the concepts of crisis, disaster, emergency management, and catastrophe interchangeably. 'Emergency' has been defined by researchers, as the disruption of the usual function of services in an organization and the environment, threatening the lives of humans and animals. However, The United Nations Office for Disaster Risk ReductionUN/ISDR (2004) mentions that emergency is more related to rehabilitation measures. A natural and man-made disaster is defined as a severe disruption to the functioning mode of society. define catastrophe as a disaster with extensive influence on the environment, humans or materials, which overwhelms the existing resources.. The concept of crisis is related to a natural or man-made situation, which may or may not threaten human life, and can occur frequently or rarely. The systematic and unsystematic risks have been explained by, who mentioned that unsystematic risk influences specific groups, while systematic risk impacts on the entire society, poses the main problem and causes disruption to infrastructure and services.

Technology growth is simultaneous with the evolution of complex information systems especially in emergency systems, which help managers to automate emergency management measures . Due to the complexity of the different kinds of disaster in different situations, and their impact on several levels of services and infrastructure, a multiparty system with special capabilities and knowledge is required for managing crisis conditions . The Geographic Information System (GIS) plays an important role in Crisis Management by storing data, managing them, analyzing, modeling and showing them on a map in digital format. An important characteristic of the GIS technology is its ability to integrate heterogeneous, Spatial, information and attribute that helps managers in decision making. Geospatial information, thematic maps and satellite image, all are mangers supporters for decision making during all phases of crisis cycle . Effectiveness and efficiency of GIS on identifying hazards, landuse, critical infrastructure, distribution of population and identifying evacuation ones have been reported by Armenakis and Nirupama (2013).

Despite the usefulness and benefits of GIS in Crisis Management, the Iran Crisis Management Organization has not adopted it; indeed, the current research examines the method to adopt GIS in Iranian Crisis Management Organizations to reduce the risks by investigating the following:

- To prioritize Personal factors in GIS adoption to reduce risks;

- To investigate interaction between Personal factors of GIS adoption in Crisis Management;

- To prioritize each Personal factor in the Crisis Management stages;

\section{Prior Studies}

Researchers on Crisis Management have identified four interrelated stages, which are: Mitigation, Preparedness, Response, and Recovery. The stable condition or incubation period is referred to as the Mitigation phase, which is generally a longterm period before the crisis. In this stage, crisis managers implement activities such 
as vulnerability assessment, increasing public awareness by education, etc. The storage of essential needs of food and medicine is vital. During this Preparedness stage, it is assumed that the Mitigation activities cannot prevent all probability of crisis; the speed of reaction to crisis for relief and rescue of injured people is a specific part of the Response stage, and because of this, this phase is also deemed to be emergency management . The Recovery stage, as the final phase in Crisis Management, refers to short-term and long-term activities to return everything (systems) to normalcy or better than before the crisis.

Because of the multidimensional nature of a crisis, different information and knowledge are required to manage it. An enormous amount of data and information that are related to various types of crises necessitates greater dependency of Crisis Management on new technology and information systems . In this regard, GIS can help the crisis manager by managing data and information on decision making for risk reduction .

However, it must be emphasized that even new automation technology cannot be a total and complete substitute for the human being and perform the entire human task . Many automation systems restructure the human tasks, such as monitoring, which requires high personality factors and knowledge to adequately carry out the new responsibilities. Therefore, researchers stress that the acquisition and implementation of information systems like GIS, are just one aspect of using this technology . To obtain full benefit and usefulness of such technological aids, personal capability, behavioral, social and culture plays an important role .

According to the Theory of Planned Behavior (TPB) and the Theory of Reasoned Action (TRA), as fundamental and basic theories on behavioral intention subjective norms and attitude are central to the person's intention to use these factors . Knowledge and experience is recognized as a factor, which leads to efficient adoption of new technology. If correctly and efficiently employed, new technology can reduce the risks in crisis management and is also much required to manage the sophisticated conditions that are posed by crisis management .

\section{Methodology}

Crisis Management is of a multidimensional character, and the purpose of this study is to provide a practical method for decision makers to adopt GIS. The study requires a specific approach to achieve the study's goal. In this regard, the study uses Analytic Network Process (ANP), a new generation of the Analytic Hierarchy Process (AHP) introduced by T.L. Saaty (1980). This method is categorized as a subset of Multiple Criteria Decision Making (MCDM), which has been found to be a most popular technique . The MCDM is an aggregation of methodologies for comparison, ranking and selecting of alternatives that are typically unequal in attributes .

Due to the AHP's weakness in analyzing the inner dependence and interaction between variables in the entire network to get feedback, Saaty introduced ANP to overcome this problem . Due to the interrelation between Crisis Management stages and interaction between Personal criteria, the current study uses the ANP method as a mature approach in the area of MCDM. The first step in the ANP method is to structure precisely the research goal. To achieve that, the study highlights the relationship and interaction between criteria and clusters, based on theories and the literature. presents the relationship and interaction between Personal criteria and Crisis management stages for GIS adoption to minimize the risks. 


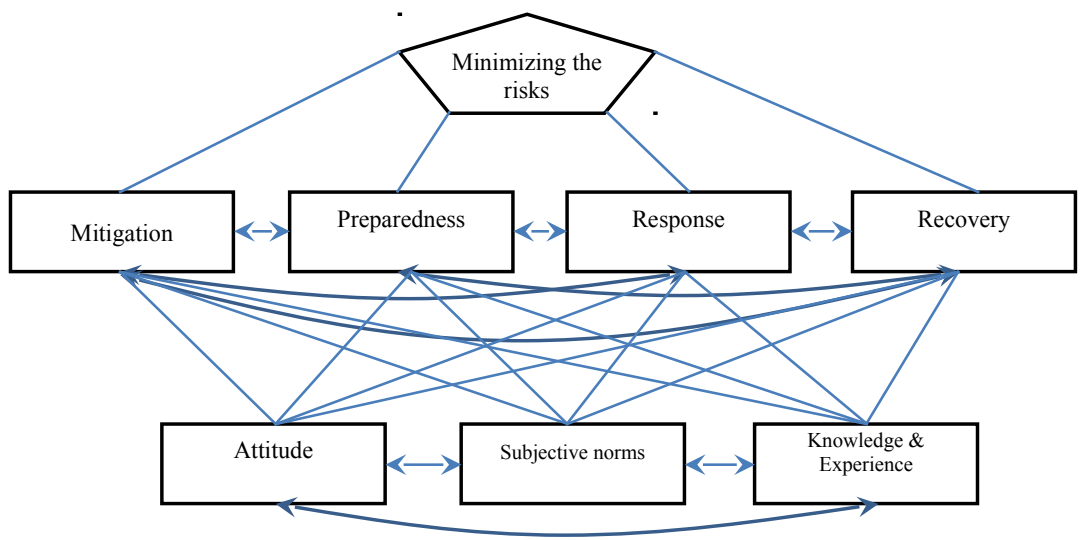

Figure 1The structured model

The second step in the ANP method is the criteria and clusters pairwise comparison to assess priorities. In this step, responders weigh and rank the criteria and clusters based on the fundamental scale of absolute numbers, introduced by T.L. Saaty (1980). Table 1 shows the explanations of each score.

The important issue in the second step is consideration of the Inconsistency Ratio, which is an index to examine the respondent consistency to compare criteria. Inconsistency below 0.1 is acceptable to many scholars . The ANP method is followed by analyzing and ranking criteria and alternatives to figure out priorities.

The study's data were gathered from Iranian experts in Crisis Management, GIS, and with at least a one-time experience in working under crisis conditions. The emphasis on expertise is because of the ANP's method that requires getting correct results .

Table 1

The fundamental scale of absolute numbers

\begin{tabular}{|l|l|l|}
\hline $\begin{array}{l}\text { Intensity of } \\
\text { importance }\end{array}$ & Definition & Explanation \\
\hline 1 & Equal Importance & Two activities contribute equally to the objective \\
\hline 3 & Moderate importance & $\begin{array}{l}\text { Experience and judgment slightly favor one activity over } \\
\text { another }\end{array}$ \\
\hline 5 & Strong importance & $\begin{array}{l}\text { Experience and judgment strongly favor one activity over } \\
\text { another }\end{array}$ \\
\hline 7 & $\begin{array}{l}\text { Very strong or } \\
\text { demonstrated importance }\end{array}$ & $\begin{array}{l}\text { An activity is favored very strongly over another; its } \\
\text { dominance is demonstrated in practice }\end{array}$ \\
\hline 9 & Extreme importance & $\begin{array}{l}\text { The evidence favoring one activity over another is of the } \\
\text { highest possible order of affirmation }\end{array}$ \\
\hline $2,4,6,8$ & $\begin{array}{l}\text { For compromise between } \\
\text { the above values }\end{array}$ & $\begin{array}{l}\text { Sometimes one needs to interpolate a compromise } \\
\text { judgment numerically because there is no good word to } \\
\text { describe it }\end{array}$ \\
\hline
\end{tabular}

\section{Results and Discussion}

Unlike the statistical research approaches, which look for relationships between variables and factors, the ANP method's output, is a kind of mathematical approach, involving the prioritization of alternatives. The selection of alternatives and 
prioritization of criteria are suitable applicable methods for academics and practitioners, particularly those looking for decision-making. Indeed, it is safe to say that ANP is beyond the statistical approaches.

Examination of the study's first objective indicates the prioritization of Personal factors on GIS adoption to reduce the risks in crisis management. The current study found that knowledge and experience (by 44.73\%) is the first criterion in GIS adoption, and Attitude (by 37.10\%) is ranked second while the Subjective norms (by $18.17 \%$ ) are a recognized as the last priority in GIS adoption in Crisis Management.

These results may be explained by the fact that the multidimensional nature of Crisis Management of varying types of crisis, at different stages, and the dynamic condition, all together require specific knowledge and experience to manage it . By the same token, the professional approach of GIS software in integrating a variety of data and information together needs professional people with particular knowledge and experience to handle the crisis. Thus, the knowledge and experience criterion plays a crucial role in the GIS adoption in Crisis Management.

The simple definition of Attitude is the personal beliefs and expectations, while the other expectations can be defined as a Subjective norms explanation. Indeed, the person believes the usefulness or perceived usefulness of GIS can lead to intention to use and actual use of this system in Crisis Management. In this way, Attitude ranks second and Subjective norms become the last priority based on the judgment of the current study respondents. Figure 2 presents the study's results.

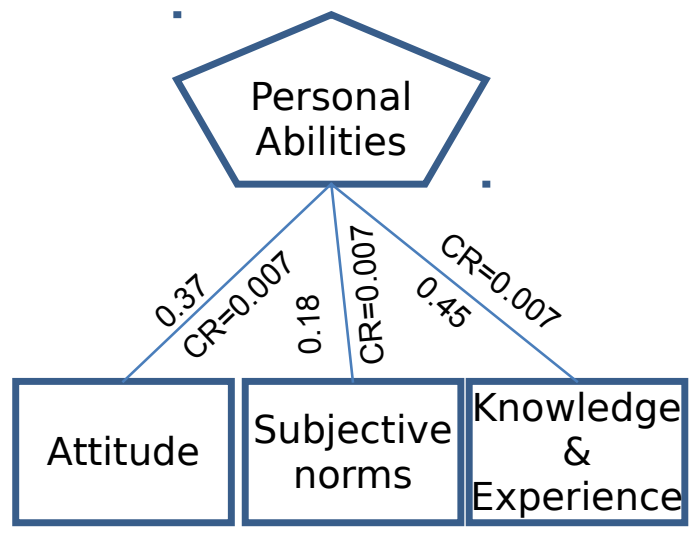

Figure 2 the Personal factors priorities

The results of investigating the interaction between Personal factors on GIS adoption are presented in Figure 3. As the table shows, when the Attitude within the GIS users is at a high level, the Knowledge and experience factor is ranked as the first (by $59.29 \%$ ) priority and Subjective norms is second (by $40.71 \%$ ) priority. In addition, even at a high level of Subjective norms, the Knowledge and experience factor is still ranked as the first (by 64.82\%) priority and Attitude becomes second (by 35.18\%). Likewise, when there is a perfect level of Knowledge and experience among users, Attitude is recognized as the first (by $66.46 \%$ ) priority and Subjective norms is ranked as the second (by 33.54\%) priority. These present results are significant for decision makers who adopt the GIS.

In Crisis Management organization, the results show that in high willingness by users and high expectation from others, Knowledge and experience plays an essential role in the GIS adoption in Crisis Management. Another significant finding is that a high 
level of Knowledge and experience among users needs perfect Attitude or willingness to use the GIS. Thus, the implementation of Human Resource (HR) activities to improve user knowledge and experience is much needed. Besides increasing Knowledge and experience, attention to Attitude elements to increase willingness to use the new technology is a crucial part of GIS adoption.

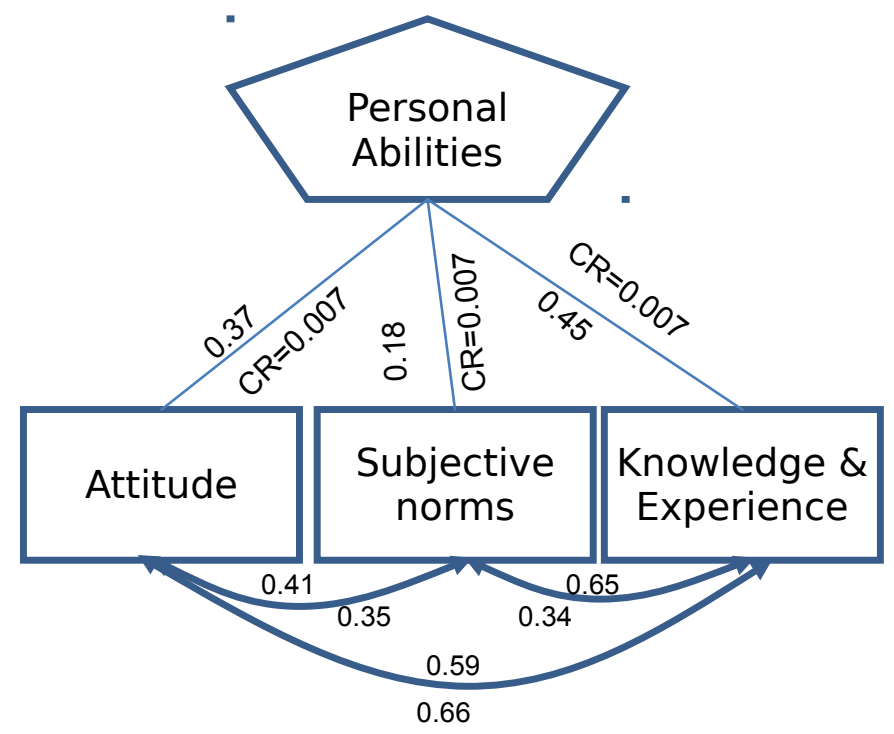

Figure 3 Interaction of personal criteria

Although Knowledge and experience, Attitude, and Subjective norms respectively are ranked first to third priorities respectively, the current study found that the Personal criteria's priorities vary from one phase of Crisis Management to another. This finding demonstrates the sophistication of Crisis management. Table 2 shows Personal criteria under different stages of Crisis Management.

Table 2 indicates Knowledge and experience is ranked as first priority in the Mitigation phase, followed by Attitude as second, and Subjective norms as the last priority. The possible explanation for this finding is related to the Mitigation phase measures. Vulnerability assessment, constructing of dams or dikes, increase in public awareness by education, etc. are some measures in the Mitigation phase, during which Knowledge and experience are important to achieve perfect management. The Mitigation phase is also recognized as a stable period, without crisis. Indeed the intention to perform some activities in this condition requires perfect Attitude, and this is the reason why the study's respondents ranked Attitude as the second priority in the Mitigation phase. As in the Mitigation phase, the study found the same priority order of Personal criteria in the Preparedness stage. Storage of essential needs of life, establishment of early warning systems, establishing some standards for emergency management and emergency maneuver are recognized as Preparedness measures in which the Knowledge and experience factor plays an essential role to achieve perfect results. Subjective norms, Attitude, Knowledge and experience respectively are ranked first, second and third priority respectively in the Response phase. The other expectation is for people to help the injured, which is a socio-cultural effect and the reason for the study's respondents to rank the Subjective norms as first priority in the Response phase, during which quick reaction is required to relieve and rescue injured people. Likewise, the Attitude of persons is important to help others, in this regard and this criterion takes second priority in the Response phase. Although a high level of Knowledge and experience is essential to help injured people, during the Response 
stage, particularly in the first $24-48$ hours, Subjective norms and Attitude play a critical role in helping to manage the crisis. The Knowledge and experience factor becomes the first priority in the recovery stage, as returning the affected area to before, or better than past requires specific Knowledge and experience. By the same token, people's expectation to solve the problem is Subjective norms that affect the Recovery stage, and Subjective norms are therefore ranked as the second priority in the Recovery phase.

Table 2

Importance of personal criteria in different stages

\section{Conclusion and Recommendation}

The number of natural and man-made disasters has increased significantly during the last decades and resulted in a growing number of people affected, together with substantial damage and cost. At the same time, there have been remarkable advances in technology, especially in Information System (IS). Indeed, investigation of factors, which can influence the use of new technology in Crisis Management, is needed. The Geographic Information System or GIS has been recognized as an important technological information system that can help in Crisis Management to reduce risks. However, it is important to realize that while new technology can enhance the quality of decision making, the role of the human being in the process is still essential and important.

The current study has also found that Knowledge and experience is an important criterion in GIS adoption, and so are Attitude and Subjective norms, which are second and third priorities in Crisis management, respectively. In addition, it should be noted that the priorities of Personal criteria change during different Crisis Management stages, which is evidence of its sophistication.

In light of the above, it is recommended that the implementation of particular Human Resource practices like improving the hiring system, and specific training for those involved should be adopted to enhance the efficiency and effectiveness of GIS adoption in Crisis Management for in the context of advanced technology, there is a need to strike a perfect relationship between man and machine, between the human capability and the technological possibilities to successful implement the GIS system in the successful management of crises.

\section{REFERENCES}


